\title{
Anabases
}

ANABASES Traditions et réceptions de l'Antiquité

13 | 2011

Varia

\section{Luciana GALLO, Lord Elgin and Ancient Greek} Architecture: The Elgin Drawings at the British Museum

\section{Amélie Perrier}

\section{OpenEdition}

Journals

Édition électronique

URL : http://journals.openedition.org/anabases/2093

DOI : 10.4000/anabases.2093

ISSN : 2256-9421

Éditeur

E.R.A.S.M.E.

Édition imprimée

Date de publication : 1 mars 2011

Pagination : 295-297

ISSN : 1774-4296

Référence électronique

Amélie Perrier, «Luciana gallo, Lord Elgin and Ancient Greek Architecture: The Elgin Drawings at the British Museum », Anabases [En ligne], 13 | 2011, mis en ligne le 01 novembre 2011, consulté le 22 septembre 2020. URL : http://journals.openedition.org/anabases/2093 ; DOI : https://doi.org/ 10.4000/anabases.2093

Ce document a été généré automatiquement le 22 septembre 2020

(c) Anabases 


\title{
Luciana GALLO, Lord Elgin and Ancient Greek Architecture: The Elgin Drawings at the British Museum
}

\author{
Amélie Perrier
}

\section{RÉFÉRENCE}

Luciana GALLO, Lord Elgin and Ancient Greek Architecture: The Elgin Drawings at the British Museum, Cambridge-New York, Cambridge University Press, 2009, 344 p. + xvii p. 150 dollars / ISBN 978-0-521-88163-0.

1 Luciana Gallo, architecte et docteur en histoire de l'architecture, propose ici une analyse de la collection conservée au British Museum des dessins réalisés entre 1799 et 1803 par les artistes au service de Lord Elgin durant son expédition en Orient. La grande qualité de l'ouvrage tient à la méthode et à l'analyse contextuelle, mais aussi à la richesse des documents présentés et aux choix de publication.

En quatre parties, l'auteur retrace l'histoire des premières expéditions en Grèce depuis le Moyen Âge, de l'expédition de Lord Elgin et de son programme artistique, enfin de la collection, avant de mener un examen critique des dessins d'architecture et de sculpture. L'ouvrage analyse les enjeux culturels, artistiques et scientifiques du projet d'Elgin. Il comprend 201 illustrations dans le texte et trois appendices importants. Le premier rassemble les transcriptions de manuscrits inédits, dont le rapport de J.I. Hittorff d'avril 1831 sur les dessins présentés par Ittar à la Société libre des beauxarts. Le deuxième présente les catalogues des dessins de l'expédition au British Museum. Le troisième propose un nouveau catalogue critique organisé topographiquement et indiquant les références des dessins, les signatures, les identifications, les techniques (aquarelle), les corrections, l'état d'achèvement et les éventuelles publications. Les notes, la bibliographie et l'index général sont rassemblés à la fin du volume. 
3 Le voyage en Grèce relève, dès le début $\mathrm{du} \mathrm{XvII}^{\mathrm{e}} \mathrm{s}$., d'une réflexion, nourrie des idées de Vitruve et des théories d'Alberti, sur un retour aux principes de l'architecture et d'une curiosité européenne pour la chute de la Grèce face aux Ottomans.

$4 \quad \mathrm{Au} \mathrm{XVIII}^{\mathrm{e}}$ s., marqué par le débat concernant la supériorité des Grecs sur les Romains, les aristocrates anglais étendent leur « Grand Tour » destiné à parachever l'éducation des jeunes gens en France et en Italie jusqu'en Grèce et en Asie Mineure où ils emmènent des artistes, non dans une intention scientifique, éducative ou éditoriale, mais pour conserver le souvenir de leur voyage. Le voyage de Stuart et Revett marque un changement, puisqu'il est prévu, dès le début, qu'il aboutisse à une importante publication. La recherche d'antiquités et la constitution de collections deviennent alors pratique courante. Les diplomates montrent un intérêt personnel en faisant copier des sculptures pour leur propre résidence. L'auteur considère cependant que l'expédition d'Elgin relève d'une autre approche et d'une autre intention que les précédentes.

5 Les objectifs artistiques sont par avance définis, ainsi qu'un programme, ce qui constitue une première originalité. Les dessins sont dès le départ destinés à la publication et des moulages de détails en sculpture et en architecture sont prévus. Mais le projet innovant d'Elgin n'est pas toujours compris ni soutenu par ses contemporains. Lord Elgin souhaite contrôler le travail de ses artistes et leur adresse un livret d'instructions. Il considère que leur production lui appartient. Alors qu'auparavant un seul artiste avait la tâche de tout dessiner, les dessins sont ici le produit d'un travail coordonné entre architectes (V. Balestra et S. Ittar), peintre de vues (G. B. Lusieri) et portraitiste (F. Ivanovitch). Les artistes ne suivent pas un ordre topographique, mais une logique stylistique, dans une démarche comparative. L. Gallo souligne l'originalité de cette entreprise en rappelant le contexte idéologique, marqué par la théorie des ricorsi de G. Vico et l'influence de ces idées sur les Lumières françaises. Bien que les théories citées soient connues (Vasari, Winckelmann, Rousseau, Montesquieu, Laugier, Burke), la rapidité de ce passage prive le propos d'une efficacité qu'une argumentation développée lui eût fait gagner.

6 L. Gallo établit la chronologie de l'expédition et des dessins qui entrent dans les collections des imprimés du British Museum en 1816.

7 L'implication de Balestra dans l'établissement des plans de Broomhall, la résidence d'Elgin, et de l'ambassade britannique à Pera laisse à Ittar le rôle majeur dans le relevé des monuments athéniens. Lord Elgin sait, en 1801, tirer parti de la situation politique favorable aux Anglais en Égypte pour ses projets artistiques, de sorte que l'étude des monuments athéniens est suivie par une mission de S. Ittar en Morée. Le 3 février 1803, Lord Elgin quitte Athènes avec les artistes qui l'accompagnent tous sauf Lusieri.

8 L. Gallo examine enfin l'intégrité de la collection en analysant les différentes sources : catalogues, correspondances, reproductions - la collection du British Museum étant incomplète. En dehors de quelques reproductions, les dessins de l'expédition n'ont jamais vraiment été étudiés. Dans son rapport sur les dessins d'Ittar, Hittorff salue les trouvailles et les progrès réalisés dans l'appréhension et la connaissance de l'architecture, en particulier du Parthénon. Le débat sur la polychromie n'est pas encore d'actualité, mais, avec Stuart, Revett, Fauvel et Chandler, Ittar est l'un des premiers à s'y intéresser. Une des plus importantes contributions de l'expédition d'Elgin, outre les corrections du plan athénien, concerne le trésor d'Atrée à Mycènes. L. Gallo souligne les qualités de la méthode d'Ittar, minutieux dans ses mesures et attentif aux données archéologiques. L'analyse montre une différence d'approche par 
rapport aux missions précédentes: il ne s'agit plus de dessiner une architecture idéalisée pour en faire un modèle à imiter, mais d'établir des comparaisons afin de saisir les variations stylistiques dans le temps et dans l'espace, en tenant compte du contexte archéologique. La méthode de représentation privilégiée des artistes d'Elgin est la coupe orthonormée. La couleur est toujours utilisée à des fins descriptives, jamais à des fins picturales. Contrairement à l'approche critique des dessins d'architecture, les dessins de sculpture, réalisés par Ivanovitch, suivent une méthode plus conventionnelle.

9 L'étude de la collection est riche d'enseignements sur le rapport des architectes du XIX siècle aux antiquités grecques et révèle l'originalité du programme d'Elgin. Si l'analyse menée par les artistes d'Elgin reste déterminée par une vision cyclique du développement de l'art grec, l'approche comparative ouvre de nouvelles questions et inaugure une méthode analytique orientée vers la technique de construction d'un édifice. Les artistes prennent conscience que les éléments sculptés ne sont pas simplement juxtaposés, mais font partie intégrante de l'édifice.

L. Gallo parle peu des marbres emportés par Elgin, ce qui suffirait à rendre son ouvrage exceptionnel, après les nombreuses publications sur le sujet (de B. F. Cook, W. St. Clair, C. Hitchens, Th. Vrettos, et en 2006, D. King). Elle met surtout l'accent sur la production scientifique et le but éducatif de l'expédition: la collection de pièces originales est effectuée et comprise dans l'intention de servir à des fins culturelles, et non pour décorer des résidences privées, comme certains contemporains l'ont reproché à Elgin. Sans vraiment entrer dans le débat, qui n'est pas l'objet de l'ouvrage, l'auteur souligne néanmoins à l'occasion - ou défend? - l'honorabilité des intentions d'Elgin, qui ne songeait pas à spolier les Grecs, mais à cultiver les Anglais.

\section{AUTEURS}

\section{AMÉLIE PERRIER}

Université Paris IV Sorbonne

École française d'Athènes

amelie.perrier@efa.gr 